\title{
Expression of the Von Hippel-Lindau Tumor Suppressor Gene, $V H L$, in Human Fetal Kidney and During Mouse Embryogenesis
}

\author{
Patricia M. Kessler,* Sandip P. Vasavada, ${ }^{*}$ \\ Raymond R. Rackley, ${ }^{\star \dagger}$ Thomas Stackhouse, ${ }^{\ddagger}$ Fuh-Mei Duh,

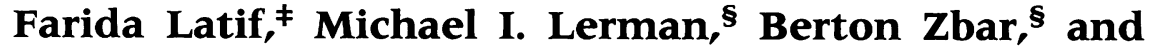 \\ Bryan R. G. Williams* \\ Departments of Cancer *Biology and ${ }^{*}$ Urology, The Cleveland Clinic \\ Foundation, Cleveland, Ohio, U.S.A. \\ ${ }^{\ddagger}$ Program Resources, Inc./DynCorp and the \\ \$Laboratory of Immunobiology, Biological Carcinogenesis and \\ Development Program, National Cancer Institute, Frederick, Maryland, \\ U.S.A.
}

\begin{abstract}
Background: Von Hippel-Lindau (VHL) disease is a familial cancer syndrome that has a dominant inherited pattern which predisposes affected individuals to a variety of tumors. The most frequent tumors are hemangioblastomas of the central nervous system and retina, renal cell carcinoma (RCC), and pheochromocytoma. The recent identification and characterization of the $V H L$ gene on human chromosome $3 p$ and mutational analyses confirms the $V H L$ gene functions as a classical tumor suppressor. Not only are mutations in this gene responsible for the VHL syndrome, but mutations are also very frequent in sporadic RCC.

Materials and Methods: $V H L$ expression in human kidney and during embryogenesis, was analyzed by in situ mRNA hybridization with ${ }^{35} \mathrm{~S}$-labeled antisense $V H L$ probes, derived from human and mouse cDNAs, on cryosections of human fetal kidney and paraffin sections of murine embryos.
\end{abstract}

Results: In human fetal kidney, there was enhanced expression of $V H L$ within the epithelial lining of the proximal tubules. During embryogenesis, VHL expression was ubiquitous in all three germ cell layers and their derivatives. Expression occurred in the cerebral cortex, midbrain, cerebellum, retina, spinal cord, and postganglionic cell bodies. All organs of the thoracic and abdominal cavities expressed $V H L$, but enhanced expression was most apparent in the epithelial components of the lung, kidney, and eye.

Conclusions: In human fetal kidney, the enhanced epithelial expression of the $V H L$ gene is consistent with the role of this gene in RCC. There is widespread expression of the $V H L$ gene during embryogenesis, but this is pronounced in areas associated with VHL phenotypes. These findings provide a histological framework for investigating the physiological role of the $V H L$ gene and as basis for further mutational analysis.

\section{INTRODUCTION}

Von Hippel-Lindau (VHL) disease is an autosomal dominant cancer syndrome characterized by the development of a variety of benign and malignant

Address correspondence and reprint requests to: Bryan R. G. Williams, Department of Cancer Biology, Research Institute, Cleveland Clinic Foundation, 9500 Euclid Avenue, Cleveland, Ohio 44195, U.S.A. tumors affecting tissues of varying embryonic origin. Affected individuals develop tumors of the central nervous system including hemangioblastomas of the spinal cord and cerebellum, retinal angiomas, pancreatic cysts, renal cell carcinomas, and pheochromocytomas. Renal cell carcinoma is the most common cause of death, with life expectancy reduced to a median of 49 years of age (1). 

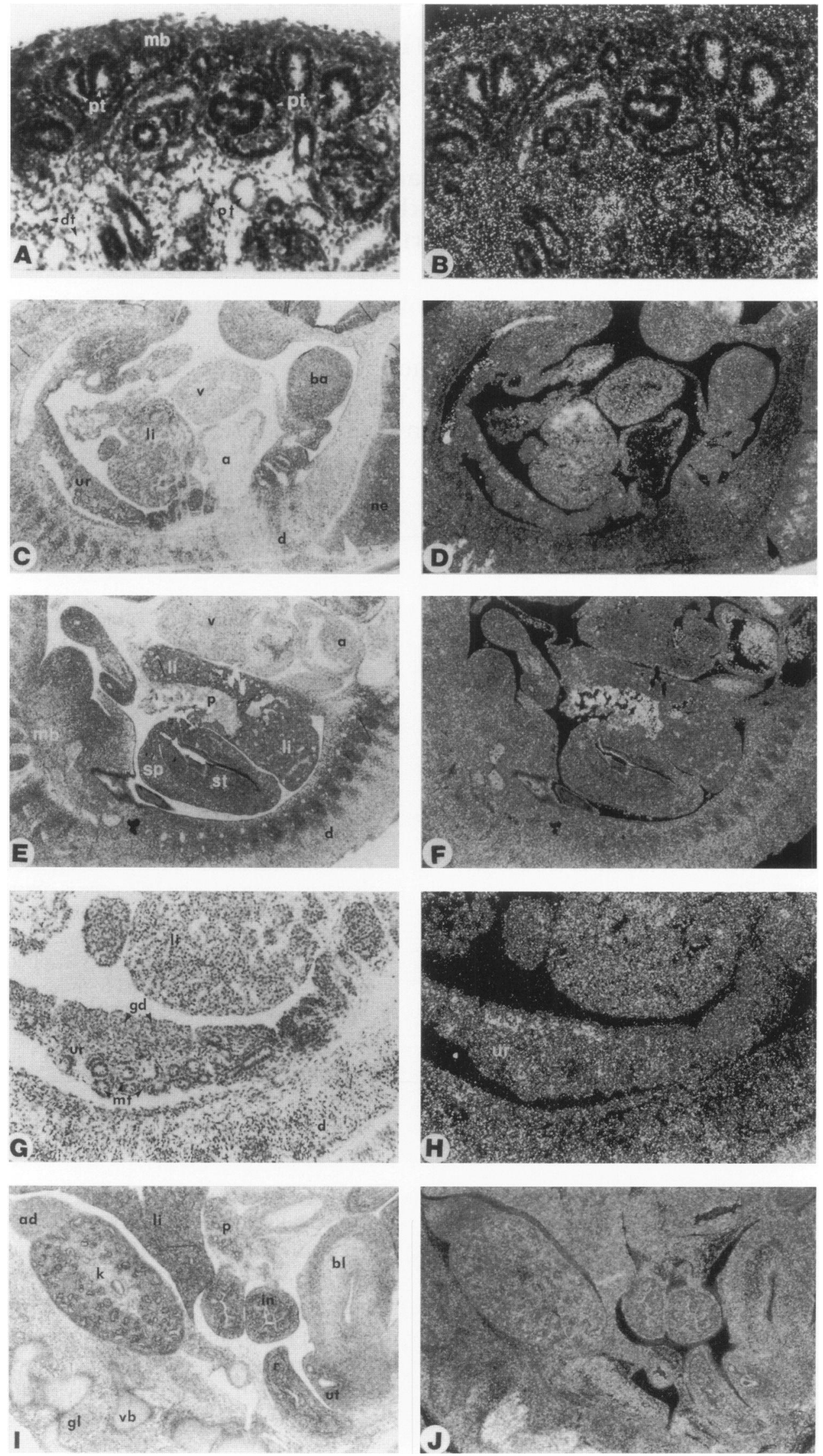
The $V H L$ tumor suppressor gene was recently isolated by positional cloning methods. Fluorescence in situ hybridization (FISH) maps the gene to a single locus at chromosome 3 p25 (2). The complete coding sequence of the $V H L$ gene has not yet been delineated, but the cloned partial cDNA encodes three exons encompassing 852 nucleotides. This sequence encodes a short open reading frame of 284 amino acids. The single copy gene is highly conserved across species as diverse as mammals, drosophila, and sea urchin, suggesting it encodes a protein with fundamental cellular function. $V H L$ is expressed in all human tissues tested by Northern analysis. Two transcripts are expressed in a spatial and temporal manner and represent alternative spliced forms of the VHL mRNA $(2,3)$.

Subsequent studies have focused on somatic mutations within the VHL tumor suppressor gene in renal cell carcinoma. RCC can be subdivided into two categories: tumors associated with or without chromosome 3 abnormalities. Most VHL and familial renal tumors as well as sporadic RCC are of the clear cell type associated with chromosome $3 p$ abnormalities $(4,5)$. Two separate investigations have recently identified VHL somatic mutations in 57 and $56 \%$ of sporadic clear cell renal carcinomas studies $(3,6,7)$. Comparison of advanced primary or metastatic renal carcinoma to localized clear cell carcinoma show that VHL mutations occur in the early stages of kidney tumorigenesis. Additionally, VHL mutations were identified in a familial RCC carrying a constitutional translocation. This form of familial cancer, as well as mutations found in both localized and advanced sporadic RCC, indicates that the VHL gene has a critical role in development of renal carcinoma (3).

In an attempt to further characterize the role of the VHL gene in differentiation and development, we have examined its expression pattern in the human fetal kidney and throughout murine embryogenesis. The VHL gene is expressed in virtually all human tissues tested by Northern hybridization, however, its exact expression pattern in regard to tissue and cellular localization remains unknown. To better define the temporal and spatial distribution of $V H L$ expression, we have performed in situ mRNA hybridization with ${ }^{35} \mathrm{~S}$-labeled antisense $V H L$ probes on frozen sections of a human fetal kidney and on paraffinembedded sections of murine embryos at different stages of development.

\section{MATERIALS AND METHODS}

\section{Embryo Collection}

Embryos of Swiss Webster matings were recovered at various stages of postimplantation development. Day 0.5 postconception was determined by the presence of a vaginal plug. Embryos were harvested on embryological days 7.5 to 17.5 , then fixed in buffered formalin and paraffin embedded. For the in situ hybridization analyses, the mouse embryos were sectioned at $4 \mu \mathrm{m}$.

\section{Human Fetal Kidney}

Human fetal kidneys were resected from a 14week-old aborted fetus and then embedded in OCT compound (Tissue Tek, Miles, Inc., Elkhart, IN, U.S.A.). For further analysis, the fetal kidneys were sectioned at $7 \mu \mathrm{m}$ on a cryostat microtome.

\section{Cloning of the Human VHL CDNA}

The g7 partial cDNA clone was isolated from a lambda gt 11 teratocarcinoma cDNA library (2).

\footnotetext{
FIG. 1. Photomicrographs of $\mathrm{VHL}$ expression in the human fetal kidney and in murine embryogenesis (A and B) Expression of $V H L$ in the human fetal kidney at 14 weeks gestation shown in toluidene blue $\mathrm{O}$-stained brightfield photomicrographs and the corresponding darkfield images. VHL expression is evident in the proximal tubules (pt), distal tubules (dt), and metanephric blastema $(\mathrm{mb})(\mathrm{A}, \mathrm{B}: \times 60)$, however, a prominent difference is noted in proximal tubular expression when compared with the other structures. (C, D, E, and F) Expression of $V H L$ in murine embryogenesis $(\mathrm{E}=13.5$ days $)$. Light and corresponding darkfield photomicrographs of thorax and abdominal development revealing expression of $V H L$ in the atrium (a) and ventricle $(v)$ of the heart. Expression is more prominent in the developing liver (li), pancreas (p), brachial arch (ba), and the urogenital ridge (ur) and cephalad, the adjacent neuroepithelium (ne) lining the developing brain structures (C, D: $\times 60 ; E, F: \times 60)$. (G, H, $\mathrm{I}$, and J) Higher magnification views of light and corresponding darkfield photomicrographs of murine VHL expression in the abdomen ( $\mathrm{E}=13.5$ days) showing moderate expression in the intestines (in), and more intense expression in the developing urogenital ridge (ur), gonad (gd), and mesonephric tubules (mt). The kidney (k) and pancreas $(p)$ show high levels of $V H L$ expression with lower levels of expression in the adjacent adrenal gland (ad), vertebral body ( $\mathrm{vb})$, ganglion ( $\mathrm{gl})$, and pelvic organs of the uterus (ut), bladder (bl), and rectum (r) (G, H: $\times 120 ; \mathrm{I}, \mathrm{J}: \times 50)$.
} 
The NotI-Xbal fragment used for the in situ analysis was subcloned from this cDNA into pBluescript II $\mathrm{KS}^{+}$(Stratagene, La Jolla, CA, U.S.A.). (Genbank Accession number L15409.)

\section{Cloning of the Mouse $V H L$ cDNA}

The Ml partial cDNA (1390 bp) was isolated from a mouse brain cDNA made in lambda ZAPII. The NotI-AccI (294 bp) fragment of the Ml clone used in in situ analysis was subcloned into pBluescript II $\mathrm{KS}^{+}$(Stratagene, La Jolla, CA, U.S.A.). The homology of the Ml VHL cDNA to the human VHL cDNA is $52 \%$ at the nucleotide level over both cDNAs. The homology between the probes used for in situ hybridization analysis is $89 \%$ at the nucleotide level. (Genbank accession number U12570.)

\section{In Situ RNA Hybridizations}

In Situ hybridizations on sections of mouse embryos as well as the human fetal kidney sections were adapted from methods previously described by Sassoon and Rosenthal and Rackley et al. $(8,9)$. The 594-bp antisense probe used for the human fetal kidney sections was generated by transcribing the NotI-XbaI fragment of the $\mathrm{g} 7$ cDNA from the T3 promoter. The sense probe of the $594 \mathrm{bp}$ was synthesized from the same NotIXbaI fragment using the T7 promoter. Both human and mouse RNA probes were labeled with ${ }^{35} \mathrm{~S}$ UTP $400 \mathrm{Ci} / \mathrm{mmole}$, Amersham Corp., Arlington Heights, IL, U.S.A.. The mouse probes were generated by transcription of the NotI-AccI fragment of the Ml cDNA from the T3 promoter to give an antisense probe of $335 \mathrm{bp}$. The sense probe was transcribed from the T7 promoter generating a 328-bp probe. RNA probes were hydrolyzed by alkali treatment to approximately 150 nt in length prior to hybridization. After hybridization at $50^{\circ} \mathrm{C}$, washing procedures were carried out as described by Wilkinson et al. (10). Following washing, the slides were dehydrated and dipped into NTB-2 emulsion (Eastman Kodak,
Rochester, NY, U.S.A.) and exposed for 2 weeks. After developing, the sections were stained with $0.5 \%$ toluidene blue $\mathrm{O}$ and mounted with Permount (Fischer Scientific, Pittsburgh, PA, U.S.A.) for observation under both brightfield and darkfield illumination using a Nikon Microphot-FXA.

\section{RESULTS \\ Localization of $V H L$ Expression During Human Fetal Nephrogenesis}

In situ localization of $V H L$ expression of 14 week gestation human kidney revealed expression throughout the renal cortex and medulla (Fig. 1 A and B). Specifically, in the renal cortex, moderate levels of $V H L$ expression were noted in the metanephric blastema. In contrast, high levels of $V H L$ expression existed within the epithelial lining of the proximal tubule. Of note, while $V H L$ expression existed in the distal convoluted tubules this expression was much less than that of the proximal tubules. Thus, while VHL expression is noted throughout stromal components of the developing kidney, there is an obvious differential expression that exists in the proximal tubules.

\section{Localization of $V H L$ Expression During Murine Abdominal and Thoracic Organogenesis}

To determine whether the $V H L$ gene is involved in specific developmental events during embryogenesis, we studied $V H L$ expression in a murine model of embryogenesis between embryologic Days 10.5 and 16.5. Following the stages of gastrulation and neurulation, the murine embryo enters the embryonic and fetal phase of organ development (11). By E 10.5 rudimentary organogenesis has progressed in a craniocaudal direction. At this stage of development, VHL expression was noted throughout the derivatives of the three germ cell layers within the pleuropericar-

FIG. 2. VHL murine thoracic and abdominal organogenesis in toluidene blue O-stained brightfield photomicrographs and corresponding darkfield images $(E=16.5$ days)

(A and B) Light and darkfield images of thorax development revealing expression in the lung (l), atrium (a), ventricle (v), and the liver (li) and vertebral body (vb) (A, B: $\times 30)$. (C-H) Higher magnification views of light and darkfield images of abdominal and pelvic organogenesis revealing low level VHL expression in the stomach (st), spleen (sp), and adrenal gland (ad), and high $V H L$ expression in the kidney (k), liver (li), testis (t), and epididymis (ep). Panels E and F demonstrate pancreatic (p) expression localizable to developing epithelial structures. Panels $G$ and $H$ reveal moderate expression in the ovary (ov) and uterus (ut) (C, D: $\times 30 ; E, F: \times 120 ; G, H: \times 100)$. 

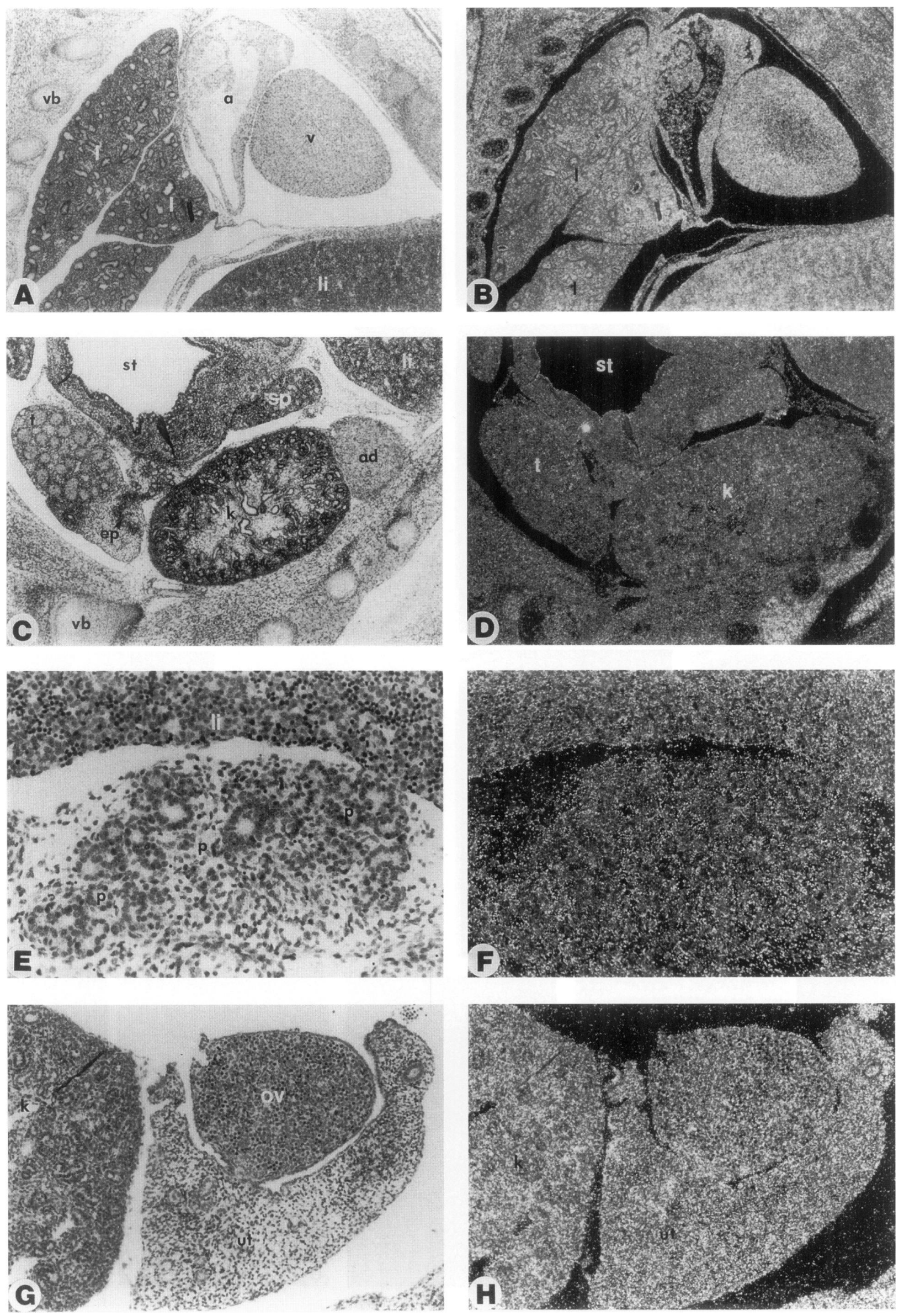

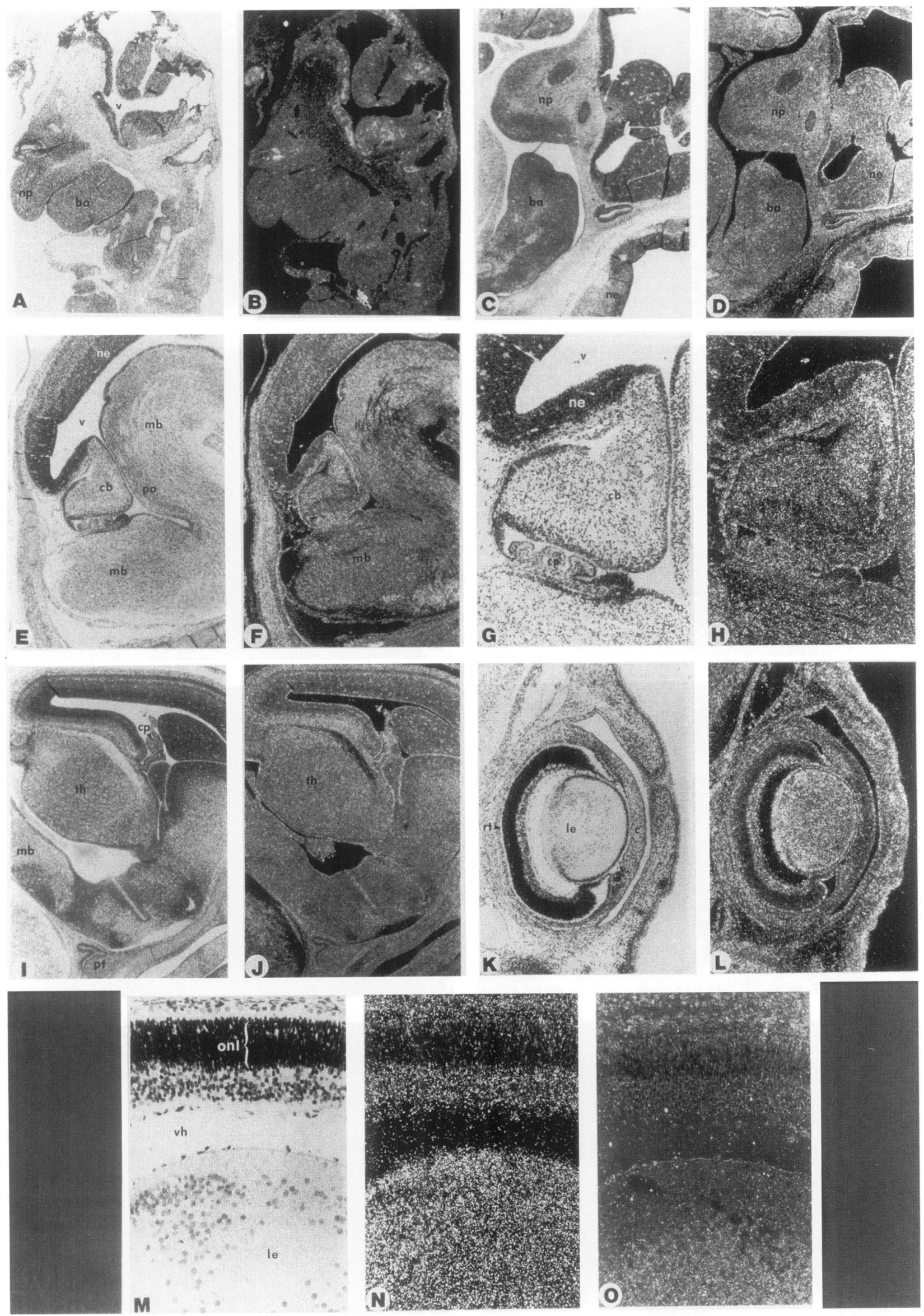
dial (thoracic) and abdominal regions (Fig. 1 $\mathrm{C}-\mathrm{J}$ ). In particular, $V H L$ expression was evident in the following structures: peripheral and central nervous system, thymus, heart, primordial lung bud, liver, spleen, stomach, pancreas, kidney, gonads, uterus, bladder, adrenal gland, intestine, somatic muscle, and cartilage (Fig. 1 C-J).

THORAX. Early in lung development, VHL expression was evident in a diffuse pattern. As the lung underwent further differentiation, a differential pattern of expression was apparent within the epithelial lining of the bronchioles. Within the developing pericardial cavity, expression of $V H L$ was noted in the pericardium and the heart musculature (Figs. 1 and 2). Minimal expression occurred within the developing thyroid gland and thymic rudiment (data not shown).

ABDOMEN. Hepatic expression of $V H L$ was diffuse in nature and with embryologic development, VHL expression appeared to be concentrated within the vascular components of the liver (Fig. $2 \mathrm{E}$ and $\mathrm{F}$ ). The $V H L$ expression noted in the primordial pancreas was unique compared with other organs since it revealed both stromal and epithelial expression as compared with the predominant epithelial expression pattern in other areas often affected in VHL disease (Figs. 1 I and $\mathrm{J}, 2 \mathrm{E}$ and $\mathrm{F}$ ). The spleen, intestine, and adrenal gland showed low $V H L$ expression. Specifically in the adrenal gland, no differential expression existed between the cortex as compared with the medulla (Figs. I I and J, $2 \mathrm{C}$ and D). This finding was interesting as the cortex is of mesodermal derivation and the medulla is derived from neural crest ectoderm.

GENITOURINARY SYSTEM. By E 10.5, the urogenital ridge was easily recognized (Fig. $1 \mathrm{C}, \mathrm{D}, \mathrm{G}$, and $\mathrm{H})$. The bilateral nephrogenic cords produce lon- gitudinal bulges referred to as urogenital ridges, which are the origins of both nephric and gonadal structures (11). Within the urogenital ridge, mesonephric structures revealed differential $V H L$ expression. The epithelial lining of the mesonephric tubules appear to express $V H L$ to a higher degree than the surrounding stromal tissue (Fig. $1 \mathrm{G}$ and $\mathrm{H}$ ). Within the caudal portion of the retroperitoneum, condensation of metanephric mesoderm around the ureteric bud signals the formation of the permanent kidney. During this early metanephric renal development no differential renal expression was noted (Fig. $1 \mathrm{E}$ and F). By E 13.5, the well-differentiated metanephric kidney revealed a differential pattern of $V H L$ expression (Figs. 1 I and J, $2 \mathrm{C}$ and $\mathrm{D}$ ). During this time period of metanephric nephrogenesis, the testis, ovary, and supporting structures reveal interesting $V H L$ expression. Specifically, the seminiferous tubules of the testis show higher $V H L$ expression as compared with the surrounding supporting stroma. In addition, the Wolffian duct derivatives consisting of the epididymis and the vas deferens all express VHL. In comparison, the ovary and Muellarian duct derivatives exhibit low levels of $V H L$ expression (Fig. $2 \mathrm{G}$ and $\mathrm{H}$ ). The developing bladder reveals minimal VHL expression.

\section{Localization of $V H L$ During Murine Nervous System Development}

The VHL expression pattern in both the central and peripheral nervous systems corroborates well with the varied clinical presentations seen in this syndrome. By E 10.5 diffuse $V H L$ expression existed within the developing nervous system. While both the neuroepithelium of the lateral ventricles and the cephalic mesenchyme exhibit $V H L$ expression, there was a higher degree of expression in the structures of the developing central nervous system (Fig. 3 A-D).

\footnotetext{
FIG. 3. VHL expression in the developing murine central nervous system (E $=16.5$ days) with light and corresponding darkfield images

(A and B) Prominent $V H L$ expression in the neuroepithelium (ne) and nasal process (np) is evident with lower levels of $V H L$ expression in the brachial arch (ba) (A, B: $\times 30)$. (C and D) Higher power views of the same structures revealing differential expression in the brachial arch (ba), neuroepithelium (ne), and nasal process (np) (C, $\mathrm{D}: \times 60)$. (E-H) Low and high power views of the midbrain $(\mathrm{mb})$, choroid plexus (cp), cerebellum (cb), pons (po), neuroepithelium (ne), and fourth ventricle $(\mathrm{v})$ demonstrating elevated levels of $V H L$ in the cerebellum and choriod plexus (cp) compared with other adjacent structures (E, F: $\times 60 ; \mathrm{G}, \mathrm{H}: \times 100)$. (I and J) Views of the mid and anterior brain demonstrating the thalamus (th), midbrain (mb), and pituitary (pt), all with minimal levels of $V H L$ expression $(\mathrm{I}, \mathrm{J}: \times 100)$. (K-O) Low and high power views of the developing eye revealing intense VHL expression in the retina (rt) and its outer neuroblastic layer (onl) in contrast to the lower levels of expression in the lens (le), vitreous humor (vh), and cornea (c). Panel O represents sense probe (K, L: $\times 125 ; \mathrm{M}, \mathrm{N}, \mathrm{O}: \times 250)$.
} 
By E 13.5, the well-developed central nervous system reveals an easily recognizable third and fourth ventricles, thalamus, midbrain, and cerebellum. In addition to the neuroepithelial $V H L$ expression in the above mentioned structures, the cerebellum and choroid plexus of the fourth ventricle appear to contain a higher degree of $V H L$ gene activity (Fig. $3 \mathrm{E}-\mathrm{H}$ ). In the peripheral nervous system, the cranial nerves of the trigeminal ganglion $(\mathrm{V})$ and the vestibulocochlear ganglion (VIII) as well as the paraspinal ganglions exhibited high levels of $V H L$ expression (data not shown).

The most impressive $V H L$ expression pattern in the central nervous system was noted in the $\mathrm{E}$ 16.5 embryo in the structures of the eye. While both the inner and outer neuroblastic layers of the retina and lens contain high $V H L$ expression, the adjacent structures reveal minimal activity. A control sense probe shows no $V H L$ expression indicating the specificity of the expression pattern (Fig. 30).

\section{DISCUSSION}

The Von Hippel-Lindau disease represents a spectrum of phakomatoses whose inherent features include those of an autosomal dominant penetrance, as well as developing lesions with a propensity towards malignant degeneration. The most common lesions in VHL include those of hemangioblastomas of the retina and cerebellum as well as angiomas of spinal cord and cystic lesions of solid organs. These cystic areas tend to affect the pancreas and epididymis and, to a lesser extent, the liver and lungs (12). Furthermore, several urologic manifestations of VHL represent a significant portion of the overall disease process and include pheochromocytoma, renal cysts, renal angiomas, and renal cell carcinomas. With the wide spectrum of anomalies in this disease process, we thought it important to further characterize the $V H L$ expression pattern throughout embryogenesis to further gain insight into normal and abnormal organogenesis, and the developmental potential of benign and malignant degeneration based on $V H L$ misregulation.

$V H L$ expression was clearly evident in all derivatives of the three germ cell layers of the murine embryo model. While specific areas known to undergo abnormal differentiation as part of the VHL syndrome revealed developmental expression of $V H L$, many areas not previously reported as developing pathologic changes in this syndrome also demonstrated VHL expression. These areas included the thymus and thyroid gland, spleen, heart, bladder, skeletal muscle, uterus, ovary, and testes.

An interesting observation apparent from this in situ investigation was the differential developmental $V H L$ expression within epithelial cells derived from mesoderm (kidney and epididymis), endoderm (lung and pancreas), and ectoderm (eye). As demonstrated in human and murine nephrogenesis, VHL expression was ubiquitous within the metanephric, ureteric, and stromal components of the developing kidney. Higher expression was noted within the welldifferentiated epithelial cells of the proximal tubules which are derived from metanephric blastema of mesodermal origin (13). This localization of VHL expression is consistent with the histopathologic studies of the origin of the clear cell phenotype of renal cell carcinoma and their associated $V H L$ mutations and/or methylation $(3,14,15)$. The developing lung also showed a higher differential expression within the endodermally derived cuboidal epithelial lining of the terminal and respiratory bronchioles. Most impressive was the VHL expression within the epithelial cells of the lens which derive from the ectoderm.

Hemangioblastomas of the central and peripheral nervous system as well as angiomas of solid organs are a central feature of the $V H L$ syndrome. While in situ expression of $V H L$ was demonstrated in the characteristic areas where these lesions develop such as the cerebellum and retina, no obvious distinctive cellular features regarding blood vessel expression were localized in these areas. The clinical development of hemangiomas and angiomas has recently been shown to be secondary to $V H L$ mutations which suggest that normal $V H L$ expression may be required for the differentiation of mesenchyme into angioblasts and endothelial cells (6).

Having characterized the pattern of $V H L$ expression throughout murine embryogenesis and human nephrogenesis, how several of the structures with high levels of $V H L$ gene expression undergo malignant transformation remains to be determined. Conversely, why some organ systems with developmental expression of $\mathrm{VHL}$ reveal no phenotypic changes in the VHL syndrome is also unclear. Renal cell carcinoma has been studied with respect to various mutations and other changes in the $V H L$ genome that may provide mechanisms for its carcinogenesis. In a 
recent study by Herman et al., they demonstrated hypermethylation of a normally unmethylated $\mathrm{CpG}$ island in the $5^{\prime}$ region of the $V H L$ gene in 5 of 26 tumors examined for this change (15). Another study on renal cell carcinomas confirmed a loss of heterozygosity ( $\mathrm{LOH}$ ) in greater than $97 \%$ of sporadic tumors with $57 \%$ having $V H L$ mutations (3). As we have shown differential expression of the $V H L$ gene in several areas of the developing embryo, it remains plausible that abnormal methylation of cytosine residues, mutations with or without LOH may convert this normal expression pattern into either a benign or malignant degenerative pattern, or may suppress functional developmental expression following terminal differentiation. Therefore, there appear to be several factors which function as initiating or regulating events of the $V H L$ gene in normal organogenesis as well as the pathogenesis of benign and malignant transformation associated with the $V H L$ syndrome.

In summary, we have characterized a unique pattern of $V H L$ expression in the human fetal kidney and murine embryo model. The differential expression patterns seen in several of the developing tissue structures corroborate with the known postnatal developmental defects that are a part of the spectrum seen in patients with von Hippel-Lindau disease but need to be confirmed by immunohistochemical localization when suitable antibodies become available. However, this work clearly provides a temporal and spatial framework for further identifying and characterizing the deleterious effects of the $V H L$ gene in a variety of disease states not previously reported in the clinical spectrum of the VHL syndrome. In addition, further investigation of the developmental regulation of the VHL gene is warranted based on the unique epithelial induction of $V H L$ expression demonstrated by this in situ hybridization analysis.

\section{NOTE}

The content of this publication does not necessarily reflect the views or policies of the Department of Health and Human Services, nor does mention of trade names, commercial products, or organizations imply endorsement by the U.S. Government. The publisher or recipient acknowledges right of the U.S. Government to retain a nonexclusive, royalty-free license in and to any copyright covering the article. Animal care was provided in accordance with the procedures outlined in the Guide for the Care and Use of Laboratory Animals (NIH Publication No. 86-23, 1985).

\section{REFERENCES}

1. Maher ER, Iselius L, Yates JR, et al. (1991) Von Hippel-Lindau disease: A genetic study. J. Med. Gen. 28: 443-447.

2. Latif F, Tory K, Gnarra J, et al. (1993) Identification of the Von Hippel-Lindau disease tumor suppressor gene. Science 260: 13171320.

3. Gnarra JR, Tory K, Weng Y, et al. (1994) Mutations of the $V H L$ tumour suppressor gene in renal carcinoma. Nature Genet. 7: 8590.

4. Farrow GM. (1989) Diseases of the kidney. In: Murphy WM (ed). Urological Pathology. W. B. Saunders, Philadelphia, pp. 409-482.

5. Kovacs G. (1989) Papillary renal cell carcinoma: A morphologic and cytogenetic study of 11 cases. Am. J. Pathol. 134: 27-34.

6. Kanno H, Kondo K, Ito S, et al. (1994) Somatic mutations of the Von Hippel-Lindau tumor suppressor gene in sporadic central nervous system hemangioblastomas. Cancer Res. 54: 4845-4847.

7. Shuin T, Kondo K, Toigoe S, et al. (1994) Frequent somatic mutations and loss of heterozygosity of the Von Hippel-Lindau tumor suppressor gene in primary human renal cell carcinomas. Cancer Res. 54: 2852-2855.

8. Sassoon D, Rosenthal N. (1993) Detection of messenger RNA by in situ hybridization. In: Wassarman PM, DePamphilis ML (eds). Gene Expression: Messenger RNA, Vol. 225, Methods in Enzymology. Academic Press, New York, pp. 384-404.

9. Rackley RR, Flenniken AM, Kuriyan NP, Kessler PM, Stoler MH, Williams BRG. (1993) Expression of the Wilms tumor suppressor gene WTl during mouse embryogenesis. Cell Growth Different. 4: 1023-1031.

10. Wilkinson DG, Bailes JA, McMahon AP. (1987) Expression of proto-oncogene int- 1 is restricted to specific neural cells in the developing mouse embryo. Cell 50: 79-88.

11. Moore KL. (1982) Genitourinary development. In: The Developing Human Embryo. W. B. Saunders, Philadelphia, pp. 40-297.

12. Christoferson LA, Gustafson MB, Petersen 
AG. (1961) von Hippel-Lindau's Disease. J.A.M.A. 178: 280.

13. Saxen L. (1987) Morphogenetic tissue interactions in the kidney. In: Organogenesis of the Kidney. Cambridge University Press, Cambridge, England, pp. 54-55.

14. Thoenes W, Storkel S, Rumpelt HJ, Moll R.
(1990) Cytomorphological typing of renal cell carcinoma-a new approach. Eur. Urol. 18(Suppl 2): 6-9.

15. Herman JG, Latif F, Weng $Y$, et al. (1994) Silencing of the $V H L$ tumor suppressor gene by DNA methylation in renal carcinoma. Proc. Natl. Acad. Sci. U.S.A. 91: 9700-9704.

Contributed by R. Sager on March 15, 1995. 